DOI: 10.17117/na.2016.02.02.405

Поступила (Received): 22.02.2016 http://ucom.ru/doc/na.2016.02.02.405.pdf

\title{
Усольцев В.К.
}

\section{Применение программного обеспечения MathCAD для синтеза регуляторов} системы автоматического управления

\author{
Usolicev V.K. \\ Using of software MathCAD for syntheses \\ regulator of the system auto control
}

Предлагается методика автоматизированного синтеза регулятора с применением программного обеспечения МathCAD. Методика заключается в автоматическом построении логарифмических частотных характеристик и запретной зоны для фазовой частотной логарифмической характеристики при заданном показателе колебательности. Методика подтверждается примерами синтеза конкретной системы управления. Рекомендуется для применения в учебном процессе и инженерной практике Ключевые слова: управление, регулятор, синтез, перерегулирование, MathCAD

\section{Усольцев Валерий Константинович}

Кандидат технических наук, доцент

Дальневосточный федеральный университет

2. Владивосток, ул. Суханова, 8

\begin{abstract}
The method of the automatic syntheses of the regulator is offered with using of software MathCAD. The Methods is concluded in automatic building of the logarithmic frequency features and forbidden of the zone for phase frequency logarithmic feature under given factor excess. The Methods is confirmed example of the syntheses concrete managerial system. It Is Recommended for using in scholastic process and engineering practical person
\end{abstract}

Key words: management, regulator, syntheses, over travel, MathCAD

\section{Usolicev Valeria Konstantinovich}

Candidate of Technical Sciences, Associate Professor Far eastern federal university

Vladivostok, Suhanova st., 8

Для обеспечения устойчивости и заданного качества переходных процессов в системе автоматического управления (САУ) с обратными связями разработан ряд методик. Наиболее распространенным является метод логарифмических частотных характеристик (ЛЧХ). Метод ЛЧХ основан на расчете логарифмической амплитудной (ЛАЧХ) и фазовой (ЛФЧХ) частотных характеристик. Метод достаточно громоздкий, требует использования множества номограмм, применим только к линейным системам. Универсальное программное обеспечение (ПО) МathCAD позволяет автоматизировать синтез регулятора известной структуры методом ЛЧХ.

Расчет и построение графиков ЛАЧХ и ЛФЧХ в ПО MathCAD [2] трудностей не вызывает, однако надо учитывать ограничения ПО MathCAD: 
1. Диапазон углов, определяемый функцией $\arg ($, лежит в диапазоне от -л до лчто позволяет определять фазовый сдвиг звеньев не выше второго порядка. Соответственно, рекомендуется объект управления и регулятор разбивать на звенья минимального порядка.

2. По MathCAD автоматически строит графики с равномерным распределением точек по оси абсцисс, что позволяет получить логарифмические графики с приемлемой точностью в диапазоне не более трех декад. Данный недостаток может быть устранен написанием собственной оригинальной программы формирования массивов для построения логарифмических графиков.

Наиболее частыми критериями, накладываемыми на качество переходного процесса САУ, являются ограничение относительного перерегулирования и быстродействие САУ при ступенчатом входном воздействии. Улучшить быстродействие САУ при фиксированном относительном перерегулировании можно только оптимальным выбором структуры регулятора. Ограничимся задачей получения заданного перерегулирования.

Важным является автоматическое формирование критерия, накладываемого на ЛАЧХ и ЛФЧХ при заданныХ критериях на переходный процесс в САУ при ступенчатом входном воздействии.

Наиболее удобной для автоматизации является методика, предложенная в [1], основанная на аналитическом определении необходимого запаса по фазе разомкнутой системы при заданном показателе колебательности замкнутой САУ. Показатель колебательности $M$ это относительный максимум амплитудночастотной характеристики замкнутой САУ. Согласно этой методике граничное значение фазового сдвига в градусах определяется уравнением

$$
\mu(\omega)=-180+\frac{180}{\pi} \cdot \arccos \left[\frac{A(\omega)^{2} \cdot\left(M^{2}-1\right)+M^{2}}{2 \cdot A(\omega) \cdot M^{2}}\right],
$$

где: $\_$- частота, $1 /$; $A(\sqsupseteq)$ - зависимость модуля передачи разомкнутой САУ от частоты; $M$ - показатель колебательности.

Методика синтеза регулятора САУ заключается в следующем:

1. Заданы передаточные функции отдельных звеньев САУ $W_{i}(s), i=1,2, \ldots, n$.

2 . Применяя замену $s \rightarrow i \cdot \omega$, получаем частотные передаточные функции звеньев САУ $W_{i}(i \cdot \omega), i=1,2, \ldots, n$.

3. Суммируем ЛАЧХ отдельных звеньев САУ $L(\omega)=\sum_{n} 20 \log \left(\left|W_{i}(\omega)\right|\right)$.

4. Суммируем ЛФЧХ отдельных звеньев СФУ $\varphi(\omega)=\sum_{n} \arg \left(W_{i}(\omega)\right)$.

5. Определяем модуль передачи разомкнутой САУ $A(\omega)=\prod_{n}\left|W_{i}(\omega)\right|$.

6. Рассчитываем граничное значение фазового сдвига $\mu(\omega)$ по формуле (1)

7. Строим ЛАЧХ $L(\omega)$, ЛФЧХ $\varphi(\omega)$ и граничное значение фазового сдвига $\mu(\omega)$ на общем графике. 
8. Подбираем параметры регулятора, добиваясь касания снизу граничным значением фазового сдвига $\mu(\omega)$ графика ЛФЧХ $\varphi(\omega)$.

Автором получена зависимость показателя колебательности от перерегулирования для САУ второго порядка, которая приведена на рисунке 1. Зависимость может использоваться и при более высоком порядке САУ.

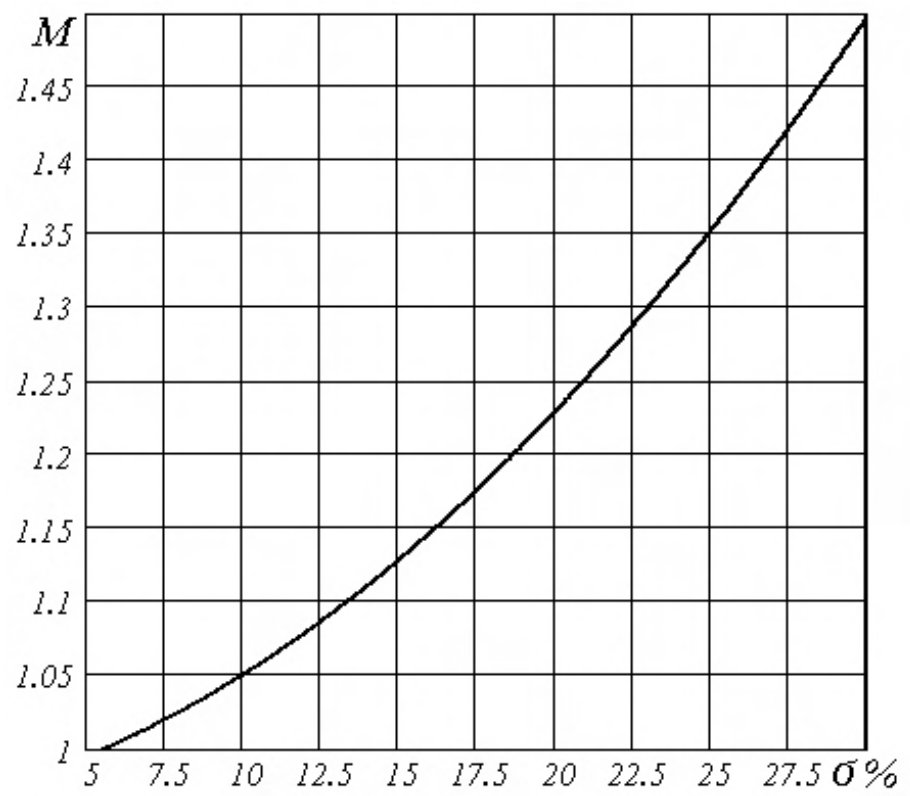

Рис. 1. Зависимость показателя колебательности от перерегулирования

Рассмотрим применение методике на примере синтеза пропорциональноинтегрального регулятора для САУ, структура которой приведена на рисунке 2.

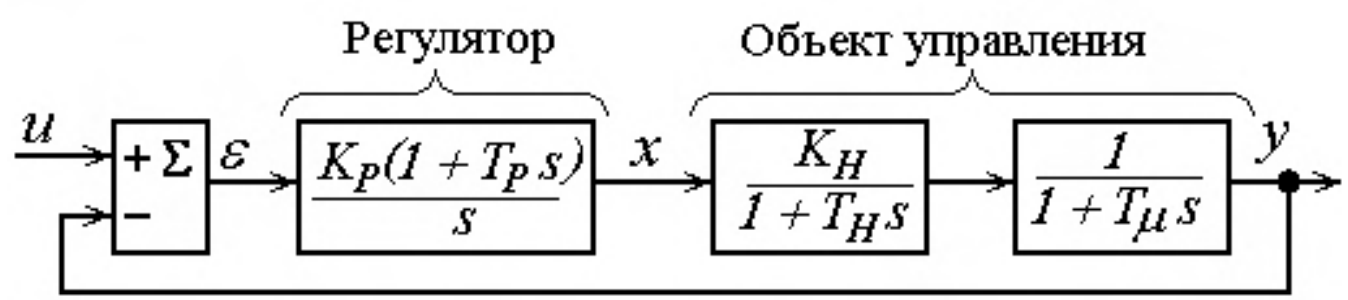

Рис. 2. Структура САУ с пропорционально-интегральным регулятором

Объект управления состоит из компенсируемого регулятором звена с передаточной функцией $W_{H}(s)=\frac{K_{H}}{1+T_{H} s}$ и некомпенсируемого звена с передаточной функцией $W_{\mu}(s)=\frac{1}{1+T_{\mu} s}$.

Передаточная функция регулятора может быть представлена в виде суммы пропорциональной и интегральной части

$$
W_{P}(s)=\frac{K_{P}\left(1+T_{P} s\right)}{s}=\frac{K_{P}}{s}+K_{P} T_{P} .
$$


Параметры объекта управления: $K_{H}:=10 T_{H}:=1.0 T_{\underline{y}}:=0.1$

Частотная передаточная функция объекта управления разбита на две части:

Компенсируемая часть $W_{H}(\omega):=\frac{K_{H}}{1+T_{H} \cdot i \cdot \omega}$

Не компенсируемая часть $W_{\mu}(\omega):=\frac{1}{1+T_{\mu} \cdot i \cdot \omega}$

Частотная передаточная функция регулятора $W_{P}(\omega):=\frac{K_{P} \cdot\left(1+T_{P} \cdot i \cdot \omega\right)}{i \cdot \omega}$

Логарифмическая амплитудно-частотная характеристика

$L(\omega):=20 \cdot\left(\log \left(\left|W_{H}(\omega)\right|\right)+\log \left(\left|W_{\mu}(\omega)\right|\right)+\log \left(\left|W_{P}(\omega)\right|\right)\right)$

Логарифмическая фазо-частотная характеристика

$\varphi(\omega):=\frac{180}{\pi}\left(\arg \left(W_{H}(\omega)\right)+\arg \left(W_{\mu}(\omega)\right)+\arg \left(W_{P}(\omega)\right)\right)$

Модуль передачи разомкнутой САУ $A(\omega):=\left|W_{H}(\omega) \cdot W_{\mu}(\omega) \cdot W_{P}(\omega)\right|$

По графику рисунка (1) выбираем $M:=1.1$, что соответствует перерегулированию $\sigma=13.5 \%$.

Граничное значение фазового сдвига

$$
\mu(\omega):=-180+\frac{180}{\pi} \cdot a \cos \left[\frac{A(\omega)^{2} \cdot\left(M^{2}-1\right)+M^{2}}{2 \cdot A(\omega) \cdot M^{2}}\right]
$$

На рисунке 3 построены графики $L(\omega), \varphi(\omega), \mu(\omega)$. Параметры регулятора подобраны так, чтобы график $\mu(\omega)$ касался снизу графика $\varphi(\omega)$. Параметры регулятора заданы как глобальные переменные, которые можно помещать в любом месте листинга.

На рисунке 4 приведены графики переходного процесса при единичном ступенчатом управляющем воздействии, полученные путем моделирования САУ в программе Multisim 11. Перерегулирование составляет $13.6 \%$, что полностью совпадает с расчетными данными.

ПО MathCAD позволяет получить модуль $A o(\omega)$ частотной передаточной функции замкнутой системы $F(\omega)$ по частотной передаточной функции разомкнутой системы $W o(\omega)$.

$$
W o(\omega):=\frac{K_{H} \cdot K_{P} \cdot\left(1+T_{P} \cdot i \cdot \omega\right)}{i \cdot \omega \cdot\left(1+T_{H} \cdot i \cdot \omega\right) \cdot\left(1+T_{\mu} \cdot i \cdot \omega\right)}, F(\omega):=\frac{W o(\omega)}{1+W o(\omega)}, A o(\omega):=|F(\omega)|
$$

График $A o(\omega)$ приведен на рисунке 5. Максимум этого графика (показатель колебательности) равен 1.1049 , что совпадает с заданным показателем колебательности.

$$
T_{P} \equiv 1, K_{p} \equiv 0.87
$$




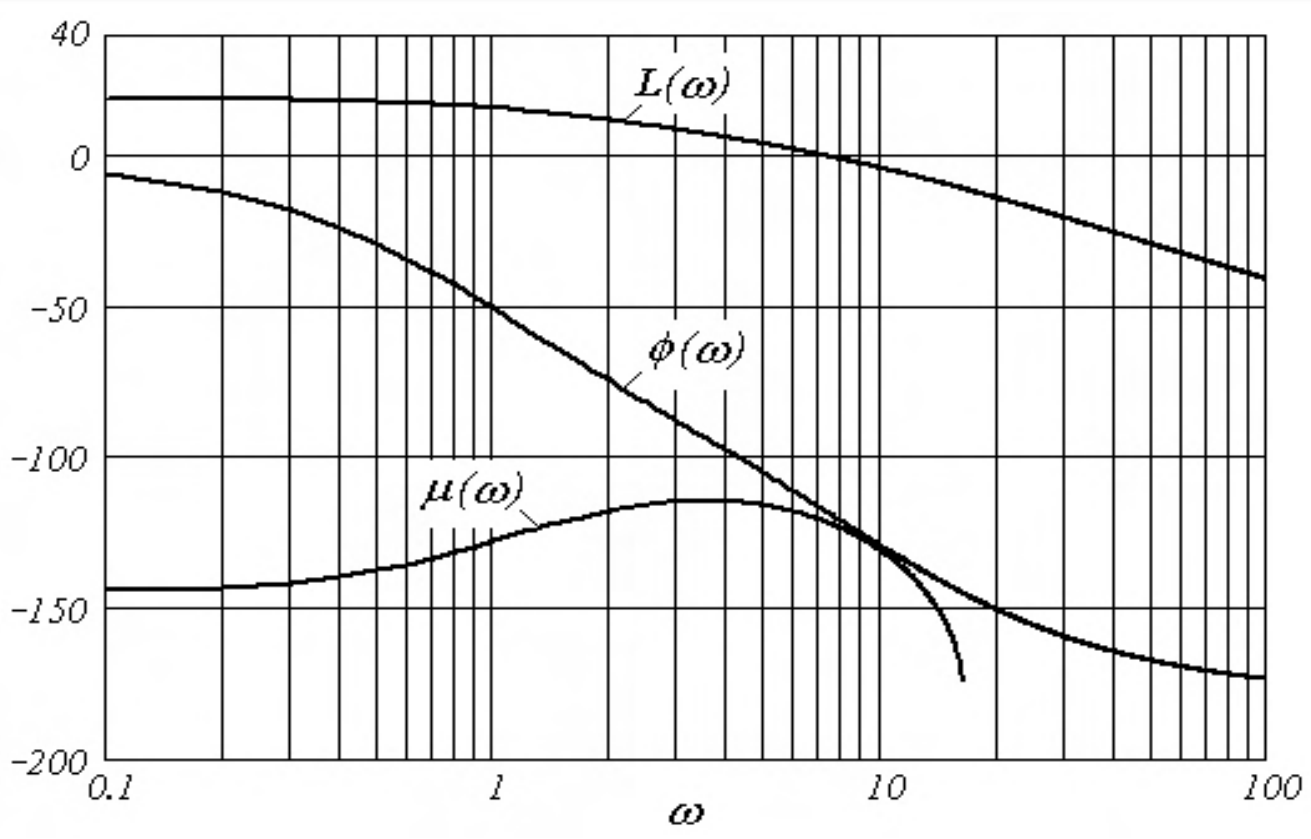

Рис. 3. Определение параметров регулятора

o.e.

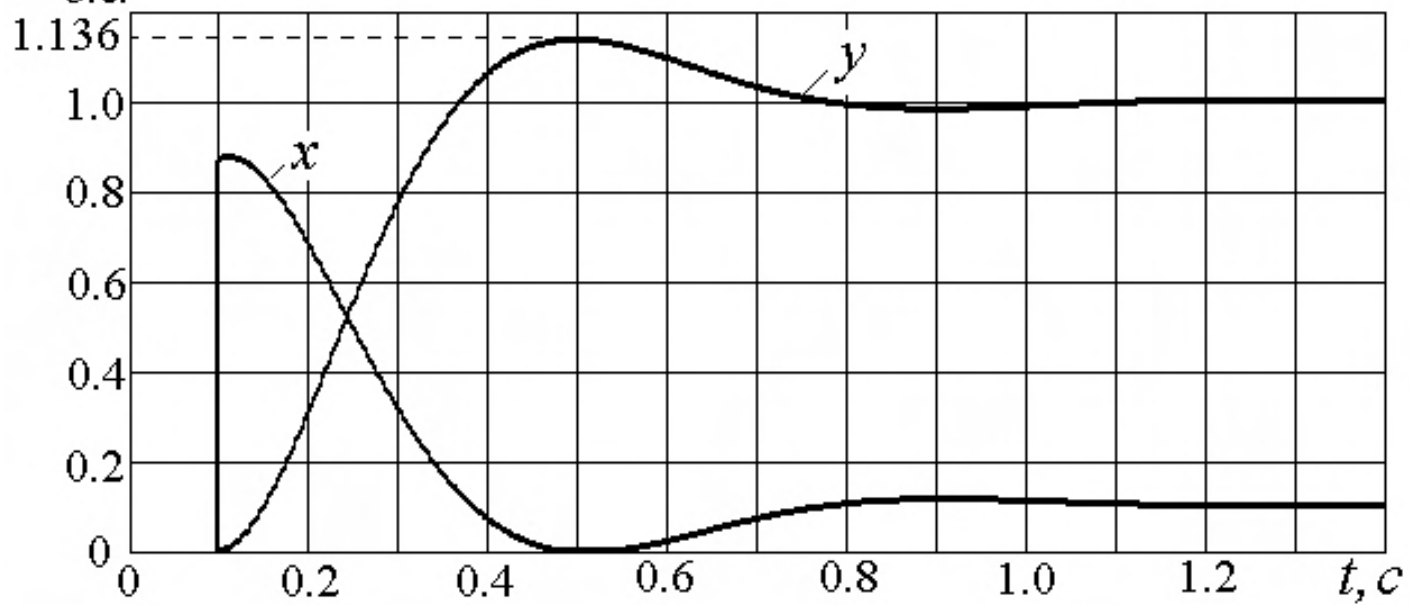

Pис. 4. Переходный процесс в САУ при $M=1.1$

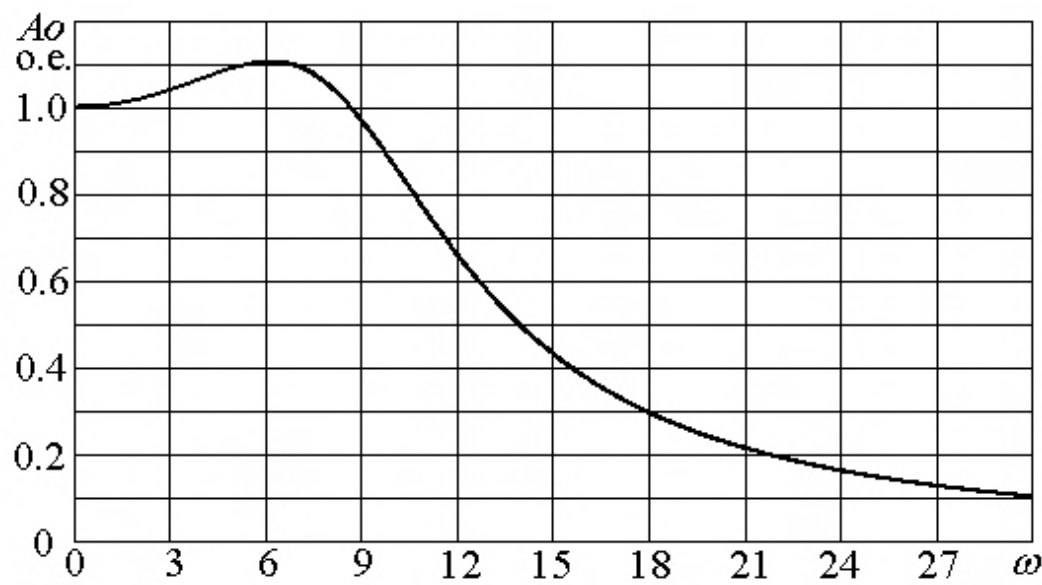

Pис. 5. Амплитудно-частотная характеристика замкнутой САУ 
Расчет и моделирование САУ при показателе колебательности $M=1.5$ сведены в таблицу 1.

Таблица 1. Параметры САУ при показателе колебательности М = 1.5

\begin{tabular}{|l|l|l|l|l|}
\hline Параметр & $M$ & $T_{P}$, с & $K_{P}$ & $\sigma \%$ \\
\hline Расчетный & 1.5 & 1.0 & 1.95 & 30 \\
\hline Экспериментальный & 1.496 & 1.0 & 1.95 & 29.9 \\
\hline
\end{tabular}

Выводы:

- ПО MathCAD позволяет автоматизировать и упростить синтез регулятора системы автоматического управления с обратными связями.

- Возможность получить частотные характеристики замкнутого контура позволяют синтезировать регуляторы многоконтурных САУ.

- Предложенная методика может использоваться как в учебном процессе, так и в инженерной практике.

\section{Список используемых источников:}

1. Бесекерский В.А., Попов Е.П. Теория систем автоматического регулирования. М., 1975,768 с.

2. Очков В.Ф. Мathcad 14 для студентов, инженеров и конструкторов. СПб.: БХВ-Петербург, 2007.368 с.

(C) 2016, Усольцев В.К.

Применение программного обеспечения МаthCAD для синтеза регуляторов системы автоматического управления
(C) 2016, Usolicev V.K.

Using of software MathCAD for syntheses regulator of the system auto control 